\title{
Ressecção completa do arco zigomático na fase de crescimento - estudo experimental em ratos
}

\section{Total resection of the zygomatic arch in the growth period - experimental study in rats}

\author{
Antônio Sílvio Fontão Procópio* \\ José Benedito Dias Lemos* \\ Elza Maria Villanova Fernandes da Rocha** \\ Alan Cruvinel Goulart***
}

\begin{abstract}
RESUMO: Foram avaliados, por meio de mensurações cefalométricas, os resultados decorrentes da ressecção unilateral total do arco zigomático em ratos Wistar. As ressecções foram realizadas com um mês de idade e os animais foram sacrificados com três meses de idade. O crânio e as hemimandíbulas foram submetidos, respectivamente às incidências radiográficas axial e lateral e, com base nestas, foram feitas mensurações por meio de um sistema de computador, comparando-se um lado com o outro, e os valores obtidos foram submetidos a análise estatística. Houve diferença significante na amplitude da fossa temporal mas não houve diferença significante para as demais mensurações da maxila. Houve diferença significante, a menor para a altura do corpo e comprimento da base da mandíbula no lado operado. UNITERMOS: Zigoma; Crescimento; Radiografia; Medidas.
\end{abstract}

\begin{abstract}
The results obtained after total unilateral resection of the zygomatic arch in Wistar rats were evaluated by means of cephalometric measurements. The resection of the right zygomatic arch was carried out in one-month-old rats, and the animals were sacrificed two months later. The skull and hemimandibles were submitted to axial and lateral radiographic incidences. Based on the obtained radiographs, measurements were carried out by means of a computer system, which compared both sides of the specimens. The obtained values were submitted to statistical analysis. There was significant difference as to the extent of the temporal fossa, but there was no significant difference as to other measures of the maxilla. There was significant difference between both sides regarding the height of the body and the length of the base of the mandible.
\end{abstract}

UNITERMS: Zygoma; Growth; Radiography; Measures.

\section{INTRODUÇÃO}

A importância do arco zigomático no desenvolvimento facial tem sido analisada e estudada por vários autores. Como sabemos, essa estrutura cresce lateral e inferiormente, tomando forma arqueada, sendo responsável pela maior largura transversal da face, tendo papel importante na simetria facial.

Lesões congênitas como disostoses mandíbulo-faciais, Sindrome de Nager, microssomia facial, Sindrome de Treacher Collins, em que o arco zigomático está parcial ou totalmente ausente, levam os pacientes a alterações no desenvolvimento facial. Outra situação de ordem não congênita que pode levar ao comprometimento parcial ou total do arco zigomático são as fraturas em pacientes jovens, não tratadas ou tratadas inadequadamente, não se esquecendo que fraturas da região zigomática apresentam alta incidência entre as fraturas da face podendo ocorrer em adultos e crianças.

A literatura tem mostrado algumas divergências em trabalhos experimentais realizados em animais. Ritsilã et al. ${ }^{7}$ (1973), Jacobs; Kronman ${ }^{4}$ (1977) e Umemura9 (1989), após ressecções parciais no arco zigomático, relatam não terem encontrado alterações significantes no desenvolvimento facial. Já Del Campo et al. ${ }^{2}$ (1995), após ressecção total uni- e bilateral do arco zigomático em ratos recém-nascidos, mostram resultados que levaram à uma assimetria facial e alterações no comprimento ântero-posterior da face, respectivamente.

Temos observado ainda na literatura que poucos trabalhos estudaram o desenvolvimento da mandíbula em animais submetidos a alterações do

*Professores Doutores; **Estagiária Didática da Disciplina de Traumatologia Maxilo-Facial; ***Mestrando em Cirurgia e Traumatologia Buco-Maxilo-Faciais - Departamento de Cirurgia, Prótese e Traumatologia Maxilo-Faciais da Faculdade de Odontologia da Universidade de São Paulo. 
Procópio ASF, Lemos JBD, Rocha EMVF da, Goulart AC. Ressecção completa do arco zigomático na fase de crescimento - estudo experimental em ratos. Pesqui Odontol Bras 2002;16(2):175-179.

arco zigomático, como fraturas, ressecção parcial e total, na fase de crescimento (Goulart et al., ${ }^{3} 1998$; Rocha et al. $\left.{ }^{8}, 1999\right)$.

O presente trabalho tem por objetivo estudar experimentalmente em ratos Wistar o crescimento facial, incluindo a mandíbula, após ressecção unilateral total do arco zigomático na fase de crescimento.

\section{MATERIAL E MÉTODOS}

Neste trabalho, foram utilizados 26 ratos Wistar com um mês de idade pesando em média $80 \mathrm{~g}$, não interessando sexo. Os animais foram submetidos à ressecção unilateral total do arco zigomático do lado direito, utilizando-se como agente anestésico o cloridrato de cetamina (Ketalar, Parke-Davis), na dose de 0,2 $\mathrm{ml} / 100 \mathrm{~g}$ de peso corpóreo, associada a xilazina (Rompum, Bayer) na dose de 0,5 ml/100 g, aplicados via intraperitoneal.

Para execução da ostectomia, foi feita uma incisão no sentido ântero-posterior, paralelamente ao arco zigomático do lado direito indo até a região pré-auricular. A seguir foi feita uma divulsão romba com desinserção total do músculo masseter no arco zigomático, permitindo exposição total deste osso, sendo a seguir realizada a ostectomia radical do mesmo, obtida com emprego de tesoura de Mayo curva. A sintese por planos foi realizada com fio de náilon monofilamentar 4.0 (Superlon, Cirumédica S/A), concluindo-se o procedimento.

No pós-operatório, os animais foram colocados em gaiolas e receberam ração granulada para roedores, fragmentada nos primeiros 15 dias, e água ad libitum. Após 60 dias do ato cirúrgico, os animais foram sacrificados e suas cabeças removidas e dissecadas, removendo-se pele e demais tecidos moles. A mandíbula foi desarticulada e as hemimandíbulas separadas na região da sínfise fibrosa.

A seguir, o crânio e as hemimandíbulas foram fixadas em formol a $10 \%$ e foram obtidas tomadas radiográficas no sentido axial para o crânio e em norma lateral para as hemimandíbulas, usando-se o aparelho de radiografia Spectro II (Dabi-Atlante) com $10 \mathrm{~mA}$ e $50 \mathrm{kV}$, na técnica do cone longo, com o objeto colocado a $10 \mathrm{~cm}$ do cone. $O$ filme usado foi o periapical (Ektaspeed, Kodak) com o tempo de exposição de $0,5 \mathrm{~s}$ para o crânio e $0,4 \mathrm{~s}$ para as hemimandibulas.

As imagens radiográficas obtidas foram digitalizadas por meio do leitor óptico (Fotovix II), utilizando-se placa de aquisição (IRIS16), por meio do pro- grama DIRACOM3, desenvolvido no Laboratório de Informática Dedicado à Odontologia (LIDO).

Nas tomadas radiográficas axiais do crânio, tanto para o lado direito (lado operado), como para o esquerdo, foram feitas as seguintes mensurações: distância entre a bula timpânica e a raiz mesial do primeiro molar superior (BT-RM); distância entre a bula timpânica e o forame infra-orbital (BT-FO); distância entre o forame infra-orbital e o ponto incisal (FO-PI); distância entre os pontos de maior concavidade na fossa temporal (sentido ântero-posterior) (FIA-FIP); distância entre a bula timpânica e o ponto de maior concavidade na região anterior da fossa temporal (BT-FIA); distância entre a bula timpânica e o ponto de maior concavidade na região posterior da fossa temporal (BT-FIP) (Figura 1).

$\mathrm{Na}$ radiografia em norma lateral para as hemimandibulas, do lado direito e esquerdo foram utilizadas as seguintes mensurações em relação à altura: distância entre o processo condilar e o processo angular da mandíbula (PC-PA); distância do ponto de intersecção da face distal do terceiro molar inferior com o ramo da mandíbula e a incisura antegônica - ponto de maior concavidade da base da mandíbula (D3M-IA); e em relação ao comprimento: distância entre a inserção do incisivo no osso e o processo angular (II-PA); distância entre a inserção do incisivo no osso e o processo condilar (II-PC) (Figura 2).

Os dados obtidos por meio destas medidas foram submetidos ao teste $t$ de Student para a verificação da significância da diferença entre os valores médios dos lados direito e esquerdo. Foi fixado o nível de significância de $5 \%(\mathrm{p} \leq 0,05)$ para todas análises estatísticas.

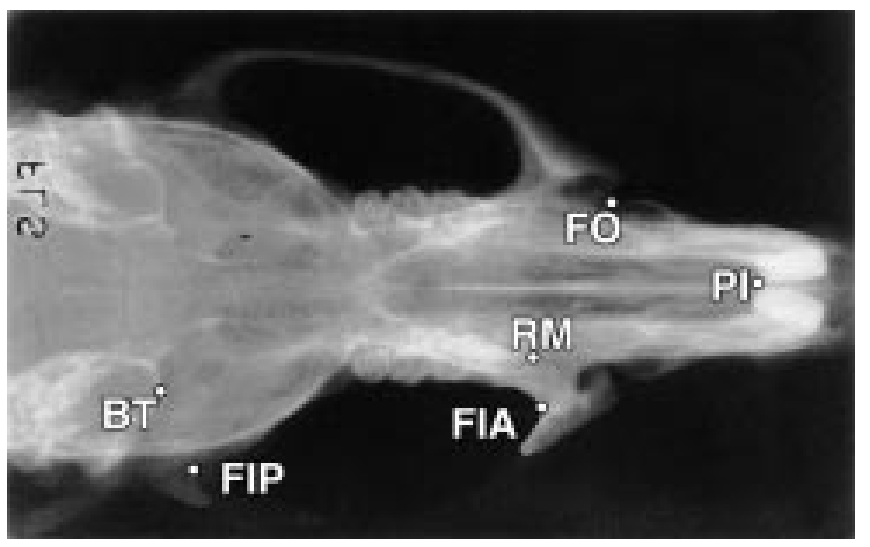

FIGURA 1 - Tomada radiográfica axial do crânio. PI = ponto incisal; $\mathrm{FO}$ = forame infra-orbitário; $\mathrm{RM}=$ raiz mesial do primeiro molar; FIA = fossa infratemporal anterior; FIP = fossa infratemporal posterior; $\mathrm{BT}=$ bula timpânica. 
Procópio ASF, Lemos JBD, Rocha EMVF da, Goulart AC. Ressecção completa do arco zigomático na fase de crescimento - estudo experimental em ratos. Pesqui Odontol Bras 2002;16(2):175-179.

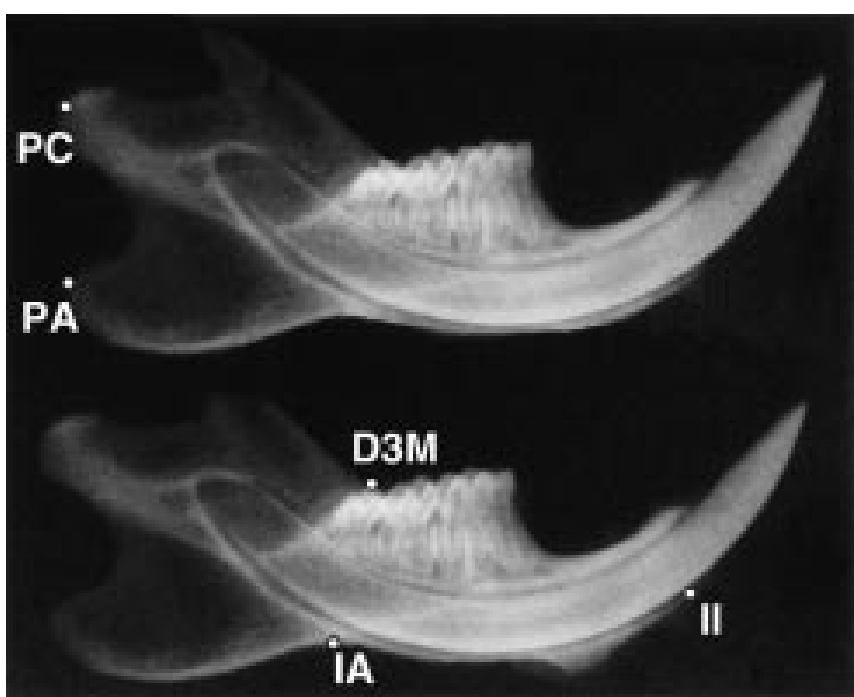

FIGURA 2 - Tomada radiográfica em norma lateral das hemimandíbulas. II = inserção do incisivo; IA = incisura antegônica; $\mathrm{D} 3 \mathrm{M}$ = face distal do terceiro molar; $\mathrm{PC}=$ processo condilar; $\mathrm{PA}=$ processo angular .

\section{RESULTADOS}

$\mathrm{Na}$ análise macroscópica, foi observada discreta assimetria facial e discreta rotação dos incisivos superiores. A seguir, são descritas as mensurações obtidas.

\section{Mensurações referentes ao terço médio da face}

\section{Distância bula timpânica-forame infra-orbital (BT-FO)}

Os valores médios da distância entre a bula timpânica e o forame infra-orbital para os lados direito e esquerdo são observados na Tabela 1. Com aplicação do teste $t$ de Student não foi constatada diferença significante.

\section{Distância bula timpânica-raiz mesial do primeiro molar (BT-RM)}

Os valores médios da distância entre a bula timpânica e a raiz mesial do primeiro molar superior para os lados direito e esquerdo são observados na Tabela 1. Com aplicação do teste $t$ de Student não foi constatada diferença significante.

\section{Distância forame infra-orbital-ponto incisal (FO-PI)}

Os valores médios da distância entre o forame infra-orbital e o ponto incisal para os lados direito e esquerdo são observados na Tabela 1. Com apli- cação do teste $t$ de Student não foi constatada diferença significante.

\section{Amplitude da fossa temporal (FIA-FIP)}

Os valores médios da distância entre os pontos de maior concavidade na fossa temporal (sentido ântero-posterior) para os lados direito e esquerdo são observados na Tabela 1. Com aplicação do teste $t$ de Student foi constatada diferença significante ao nível de $5 \%$.

\section{Distância bula timpânica-parte anterior da fossa temporal (BT-FIA)}

Os valores médios da distância entre a bula timpânica e o ponto de maior concavidade na região anterior da fossa temporal para os lados direito e esquerdo são observados na Tabela 1. Com aplicação do teste $t$ de Student foi constatada diferença significante ao nivel de $1 \%$.

\section{Distância bula timpânica-parte posterior da fossa temporal (BT-FIP)}

Os valores médios da distância entre a bula timpânica e o ponto de maior concavidade na região posterior da fossa temporal para os lados direito e esquerdo são observados na Tabela 1. Com aplicação do teste $t$ de Student foi constatada diferença significante ao nivel de $1 \%$.

\section{Mensurações referentes à mandíbula Distância processo condilar-processo angular (PC-PA)}

Os valores médios da distância entre o processo condilar e o processo angular da mandíbula para os lados direito e esquerdo são observados na Tabela 2. Com aplicação do teste $t$ de Student não foi constatada diferença significante.

TABELA 1 - Mensurações obtidas na incidência radiográfica axial do crânio seco. Valores em milímetros.

\begin{tabular}{l|r|r|r|c}
\hline \multirow{2}{*}{ Mensuração } & \multicolumn{2}{|c|}{ Experimental } & \multicolumn{2}{c}{ Controle } \\
\cline { 2 - 5 } & Média & dp & Média & dp \\
\hline BT-FO & 17,47 & 0,82 & 17,45 & 0,80 \\
BT-RM & 20,84 & 0,78 & 21,40 & 0,88 \\
FO-PI & 8,55 & 0,70 & 8,52 & 0,47 \\
FIA-FIP** & 15,48 & 0,79 & 14,53 & 0,62 \\
BT-FIA* & 17,25 & 0,78 & 16,93 & 0,84 \\
BT-FIP* & 5,04 & 0,33 & 5,54 & 0,38 \\
\hline \hline
\end{tabular}

${ }^{*} \mathrm{p} \leq 0,01 ;{ }^{* *} \mathrm{p} \leq 0,05$. 
Procópio ASF, Lemos JBD, Rocha EMVF da, Goulart AC. Ressecção completa do arco zigomático na fase de crescimento - estudo experimental em ratos. Pesqui Odontol Bras 2002;16(2):175-179.

TABELA 2 - Mensurações obtidas na incidência radiográfica lateral das hemimandíbulas. Valores em milimetros.

\begin{tabular}{l|r|c|c|c}
\hline \multirow{2}{*}{ Mensuração } & \multicolumn{2}{|c|}{ Experimental } & \multicolumn{2}{c}{ Controle } \\
\cline { 2 - 5 } & Média & $\mathrm{dp}$ & Média & $\mathrm{dp}$ \\
\hline PC-PA & 8,24 & 0,54 & 8,22 & 0,47 \\
D3M-IA* & 6,14 & 0,26 & 6,36 & 0,21 \\
II-PA** & 22,89 & 1,34 & 23,75 & 1,23 \\
II-PC & 24,11 & 1,08 & 24,29 & 0,87 \\
\hline \hline
\end{tabular}

${ }^{*} \mathrm{p} \leq 0,01 ;{ }^{* *} \mathrm{p} \leq 0,05$.

\section{Distância face distal do terceiro molar-incisura antegônica (D3M-IA)}

Os valores médios da distância entre o ponto de intersecção da face distal do terceiro molar inferior com o ramo ascendente e a incisura antegônica para os lados direito e esquerdo são observados na Tabela 2. Com aplicação do teste $t$ de Student foi constatada diferença significante ao nivel de $1 \%$.

\section{Distância inserção do incisivo-processo angular (II-PA)}

Os valores médios da distância entre a inserção do incisivo no osso e o processo angular para os lados direito e esquerdo são observados na Tabela 2. Com aplicação do teste $t$ de Student foi constatada diferença significante ao nível de $5 \%$.

\section{Distância inserção do incisivo-processo condilar (II-PC)}

Os valores médios da distância entre a inserção do incisivo no osso e o processo condilar para os lados direito e esquerdo são observados na Tabela 2. Com aplicação do teste $t$ de Student não foi constatada diferença significante.

\section{DISCUSSÃO}

Os resultados obtidos mostram que, no terço médio da face, do lado operado, houve diferenças significantes - a maior, para a distância entre a bula timpânica e a concavidade anterior da fossa temporal; a menor para a distância entre a bula timpânica e a cavidade posterior da fossa temporal; e a maior para a distância entre concavidade anterior e concavidade posterior da fossa temporal no lado operado. Esses resultados mostram que houve, no lado operado, alongamento no sentido ântero-posterior da fossa temporal. Como entre as demais medidas do terço médio da face não houve diferenças significantes entre os dois lados, podemos dizer que, em nosso trabalho, com a metodologia aplicada, não houve alteração no sentido ântero-posterior na região anterior da face. Esses resultados nos levam a concordar em parte com o trabalho de Jacobs; Kronman ${ }^{4}$ (1977) que realizaram ostectomia parcial em ratos usando metodologia semelhante e afirmaram que, quando somente um arco zigomático é seccionado, a raiz posterior (processo zigomático do osso temporal) do lado seccionado parece se deslocar para a região posterior. Nossos resultados mostram que não só o processo zigomático do temporal se deslocou, como também o processo zigomático da maxila, promovendo um aumento da fossa temporal no sentido ântero-posterior. Provavelmente, a ausência da "cinta muscular" representada pelo músculo masseter, desinserido do arco zigomático durante o ato operatório, pode ser a responsável pela abertura da fossa temporal do lado operado. Outra possibilidade é a descontinuidade do arco, perdendo a capacidade de manter próximas suas inserções, alongando a fossa.

Não observamos assimetria facial importante e nem alterações oclusais foram notadas. Os resultados são semelhantes aos obtidos por Umemura ${ }^{9}$ (1989) que realizou ressecção parcial do arco zigomático em ratos, afirmando que alterações oclusais e assimetrias ocorreram somente em animais com 120 dias de pós-operatório. Em nosso trabalho, os animais foram sacrificados com 60 dias de pós-operatório.

Del Campo et al. ${ }^{2}$ (1995) demonstraram em seu trabalho um aumento da projeção facial e diminuição da largura da face após a ressecção total do arco zigomático em animais recém-nascidos, com um dia de idade, no lado operado. Provavelmente, isto se deveu à "cinta muscular" que não bloqueou o crescimento ósseo, e também não estimulou o crescimento nos pontos de inserção. No entanto, neste trabalho foram utilizados ratos com um mês de idade que tiveram a possibilidade de receber os estímulos dos músculos masseter e temporal.

Na mandibula, obtivemos diferença significante menor na distância entre a intersecção da face distal do terceiro molar com o ramo da mandíbula e a incisura antegônica, mostrando uma diminuição em altura na região de corpo de mandíbula no lado operado. A distância entre o processo condilar e o processo angular não se alterou significantemente, mostrando que, na porção posterior da mandíbula, não houve diminuição de altura em ambos os 
Procópio ASF, Lemos JBD, Rocha EMVF da, Goulart AC. Ressecção completa do arco zigomático na fase de crescimento - estudo experimental em ratos. Pesqui Odontol Bras 2002;16(2):175-179.

lados. Portanto, a perda em altura se deu na região de corpo no lado operado, evidenciando que essa região, em termos de altura, foi a mais afetada com a perda da inserção do masseter na área mais central do arco zigomático.

Provavelmente, a região posterior da mandíbula manteve o crescimento em altura, devido à inserção de algumas fibras do músculo masseter no processo zigomático do temporal, permitindo o desenvolvimento dessa região. Rocha et al. ${ }^{8}$ (1999), após fratura unilateral do arco zigomático, encontraram diminuição em altura em toda a mandíbula no lado operado. Segundo esses autores, danos às fibras do músculo temporal promovidas pela fratura foram responsáveis por tais resultados.

Em relação ao comprimento da mandíbula segundo os dados colhidos, tivemos um encurtamento da base, com a diminuição da distância entre a inserção do incisivo e o processo angular do lado operado. A distância entre esta e o processo condilar não foi alterada. Poderíamos explicar tais fatos devido a essas regiões apresentarem inserções musculares diferentes. Enquanto a base da mandíbula, que recebe inserção do músculo masseter e devido à ressecção do arco zigomático sofreu a desinserção do mesmo, teve diminuído o es-

\section{REFERÊNCIAS}

1. Crivello Junior O, Luz JGC, Lemos JBD, Rezende JRV. Considerations statistiques sur le fractures isolées maxillofaciales a São Paulo. Rev Stomat Chir Maxillofac 1989;90:100-3.

2. Del Campo AF, Elizondo MM, Magnelli LM, Valadez AS, Outiveros DS. Craniofacial development in rats with early resection of the zygomatic arch. Plast Reconstr Surg 1995;95:486-95.

3. Goulart AC, Fernandes EMV, Cavalcanti FC, Novelli MD, Luz JGC. Fratura do arco zigomático no período de crescimento: estudo experimental em ratos. Rev Odontol Univ São Paulo 1998;12:75-80.

4. Jacobs B, Kronman JH. The zygomatic arch and its possible influence on craniofacial growth and development. Angle Orthodont 1997;47:136-40.

5. Luz JGC, Yamamoto MK, Crivello Junior O, Procópio ASF. Transfixação do fio de Kirschner no tratamento de fraturas tímulo ao crescimento, o processo condilar não sofreu qualquer alteração e pode continuar o seu processo normal de crescimento.

\section{CONCLUSÕES}

Pelos resultados obtidos por meio da metodologia aplicada, podemos concluir que:

- houve aumento significante da amplitude da fossa temporal no sentido ântero-posterior no lado operado, sem alteração na região anterior da face como um todo;

- não houve desvio significante da linha média no terço médio da face;

- houve perda em altura na região de corpo de mandíbula sem alteração na região posterior, no lado operado;

- houve diminuição do comprimento da base de mandíbula, no lado operado;

- houve importante deslocamento do processo zigomático do temporal para a região posterior, no lado operado.

\section{AGRADECIMENTO}

Ao Professor Doutor João Gualberto de Cerqueira Luz, pelo auxílio laboratorial.

instáveis no complexo zigomático. Rev Odontol Univ São Paulo 1993;7:279-84.

6. Palma VC, Luz JGC, Correia FAS. Freqüência de fraturas faciais em pacientes atendidos num serviço hospitalar. Rev Odontol Univ São Paulo 1995;9:121-6.

7. Ritsilã V, Alhopuro S, Ranta R. The role of the zygomatic arch in the growth of the skull in rabbits. Proc Finn Dent Soc 1973;69:164-7.

8. Rocha EMVF, Goulart AC, Novelli MD, Luz JGC. Efeitos da fratura do arco zigomático no crescimento facial em ratos jovens. Rev Odontol Univ São Paulo 1999;13:37-41.

9. Umemura S. Experimental study of the influence of removal of the zygomatic arch on craniofacial growth and development. Shikwa Gakuho 1989;89:981-1019.

10. Vargervik KL, Farias M, Ousterhout D. Changes in zygomatic arch position following experimental lateral displacement. J Craniomaxillofac Surg 1987;15:208-12. 